\title{
Alternative inert gas washout outcomes in patients with primary ciliary dyskinesia
}

\author{
Sylvia Nyilas ${ }^{1,2,6}$, Anne Schlegtendal ${ }^{3,6}$, Florian Singer ${ }^{1,4}$, Myrofora Goutaki $^{1,5}$, \\ Claudia E. Kuehni ${ }^{5}$, Carmen Casaulta ${ }^{1}$, Philipp Latzin ${ }^{1,2,7}$ and \\ Cordula Koerner-Rettberg ${ }^{3,7}$
}

Affiliations: ${ }^{1}$ Paediatric Respiratory Medicine, Dept of Paediatrics, Inselspital, Bern University Hospital, University of Bern, Switzerland. 'Dept of Paediatric Pulmonology, University Children's Hospital Basel (UKBB), Basel, Switzerland. ${ }^{3}$ Dept of Paediatric Pulmonology, University Children's Hospital of Ruhr University Bochum at St. Josef-Hospital, Bochum, Germany. ${ }^{4}$ Division of Respiratory Medicine, University Children's Hospital Zurich, Zurich, Switzerland. ${ }^{5}$ Institute for Social and Preventive Medicine, University of Bern, Bern, Switzerland. ${ }^{6}$ Contributed equally as first authors. ${ }^{7}$ Contributed equally as senior authors.

Correspondence: Cordula Koerner-Rettberg, University Children's Hospital Bochum, Alexandrinenstraße 5, 44791 Bochum, Germany. E-mail: Cordula.Koerner-Rettbergarub.de

@ERSpublications

Alternative washout outcomes of shorter test duration detect ventilation inhomogeneity in patients with PCD http://ow.ly/ffnL307OzWX

Cite this article as: Nyilas S, Schlegtendal A, Singer F, et al. Alternative inert gas washout outcomes in patients with primary ciliary dyskinesia. Eur Respir J 2017; 49: 1600466 [https://doi.org/10.1183/ 13993003.00466-2016].

ABSTRACT The lung clearance index (LCI) derived from a nitrogen multiple breath washout test $\left(\mathrm{N}_{2}-\mathrm{MBW}\right)$ is a promising tool to assess small airways disease in primary ciliary dyskinesia, but it is difficult to apply in routine clinical settings because of its long measuring time. In this study, we aimed to assess alternative indices derived from shorter washout protocols.

49 patients with primary ciliary dyskinesia (mean age $14.7 \pm 6.6$ years) and 37 controls (mean age $14.3 \pm 1.4$ years) performed $\mathrm{N}_{2}-\mathrm{MBW}$ and double-tracer gas (DTG) single-breath washout tests. Global (LCI and moment ratio $\left.\left(\mathrm{M}_{2} / \mathrm{M} 0\right)\right)$ ), conductive (Scond) and acinar ventilation inhomogeneity (DTG Slope III (SIII-DTG)) were determined for each individual. The main outcomes were 1) the ability to detect abnormal lung function from washout indices (>1.64 z-scores) and 2) measurement duration.

The prevalence of abnormal values for LCI $2.5 \%$ was 37 out of 47 (79\%), for LCI5\% was 34 out of 47 (72\%), for M2/M0 was 34 out of 47 (72\%), for Scond was 36 out of 46 (78\%) and for SIII-DTG was 12 out of 35 (34\%). Mean \pm SD duration of measurement was $19.8 \pm 11.2 \mathrm{~min}$ for LCI $2.5 \%, 10.8 \pm 4.6 \mathrm{~min}$ for LCI5\% and 8.6 $\pm 2.3 \mathrm{~min}$ for Scond.

Compared to standard LCI $2.5 \%$, ventilation inhomogeneity was detected by LCI5\%, moment ratio and Scond with comparable sensitivity while measurement duration was significantly shorter. Longitudinal studies will show which outcome is most suitable and practical in terms of sensitivity, duration and variability within the course of primary ciliary dyskinesia lung disease.

This article has supplementary material available from erj.ersjournals.com

Support statement: The work for this report was funded by the Fondation Botnar and the Linde Group. The funders had no role in study design, data collection and analysis, decision to publish, or preparation of the manuscript. Funding information for this article has been deposited with the Open Funder Registry.

Conflict of interest: None declared.

Copyright @ERS 2017 


\section{Introduction}

Patients with primary ciliary dyskinesia (PCD) suffer from recurrent lower respiratory tract infections and mucus plugging, ultimately leading to bronchiectasis [1]. As in cystic fibrosis (CF), abnormal peripheral ventilation is more common in patients with PCD than impaired central airway ventilation, and many PCD patients show functional impairment in their peripheral ventilation even with normal central airway function [2,3]. Despite this, lung function in patients with PCD is usually monitored by spirometry, which mainly measures central obstruction. In 2009, a European Respiratory Society (ERS) consensus statement emphasised the need to improve the sensitivity of lung function testing in patients with PCD [4]. As in CF, several studies in PCD have suggested that inert gas washout testing might be better able to detect the early stages of impaired lung function than spirometry. The lung clearance index (LCI2.5\%) is derived from a nitrogen multiple breath washout test $\left(\mathrm{N}_{2}-\mathrm{MBW}\right)$ and is calculated as the lung volume turnovers required to reach $1 / 40$ th of the starting $\mathrm{N}_{2}$ concentration. Using LCI $2.5 \%$ to estimate global ventilation inhomogeneity (VI) showed a good association with the structural lung damage detected by high-resolution computed tomography (HRCT) in children mildly affected by PCD [2, 3]. A drawback of the LCI measurement is its long washout time in severely affected patients, hampering its implementation in routine clinical practice [5].

In consideration of this, shorter gas washout protocols, such as the LCI5\% [5-7] and a new tidal single breath washout test (SBW) based on helium and sulfur hexafluoride (double-tracer gas (DTG)), seem promising to shorten the assessment of VI and to provide more specific estimates as to where in the airway tree VI may originate. These outcome variables are sensitive markers to detect diffusion- and diffusion-convection-dependent VI in lung diseases such as CF, asthma and chronic obstructive pulmonary disease (COPD) [8-13]. In patients with PCD, the diagnostic performance of shorter gas washout protocols is currently unknown.

The aim of the study was to examine the ability of alternative gas washout variables to detect abnormal lung function in comparison with LCI in patients with PCD. In a second step, we assessed the measurement duration of the alternative gas washout protocols.

\section{Material and methods}

\section{Study design}

This was a cross-sectional observational multicentre study. Recruitment took place between March 2013 and April 2015 in two outpatient clinics. Patients and healthy controls underwent $\mathrm{N}_{2}-\mathrm{MBW}$ followed by DTG-SBW on the same day. Only patients with PCD performed additional spirometry.

\section{Subjects}

We enrolled 49 paediatric and adult patients with PCD, aged (mean \pm SD) $14.7 \pm 6.6$ years. Patients were enrolled at the University Children's Hospital of Ruhr, University Bochum, St Josef Hospital, Germany $(n=46)$ and at the University Children's Hospital of Bern, Switzerland $(n=3)$. Healthy controls $(n=37)$, aged 14.3 \pm 1.4 years, were recruited from the surgical outpatient clinic at the Children's Hospital of Bern. All patients with PCD had a confirmed clinical, functional and (if applicable) structural PCD diagnosis assessed by cilia motility and structure in nasal brushing samples according to guidelines $[4,14,15]$. PCD diagnosis relied on at least two congruent pathological results on repetitive high-frequency-video microscopy together with relevant pathology in either transmission electron microscopy, immunofluorescence or genetics. Further details on patient characteristics are provided in table 1 and in the supplementary material and tables E1, E2 and E3. None of the controls had asthma or chronic lung disease and none of the subjects had signs of acute respiratory tract infection or acute pulmonary exacerbation (marked increase in cough, change in sputum amount or colour, fever, or malaise) at the time of measurement. The study was approved by the Ethics Committee of the Ruhr University of Bochum and of the Canton of Bern. We obtained written informed consent from parents or participants older than 18 years, as well as written assent from all participating children and adolescents.

\section{Lung function assessment}

Nitrogen multiple breath washout

All subjects performed two different tidal gas washout measurements, triplicate $\mathrm{N}_{2}-\mathrm{MBW}$ and DTG-SBW, with a commercially available device (Exhalyzer D, Eco Medics AG, Duernten, Switzerland) [16] according to consensus $[6,17]$. From $\mathrm{N}_{2}-\mathrm{MBW}$ the following indices were assessed: LCI2.5\%, LCI5\%, Scond, Scond ${ }^{*}$, Sacin, $\mathrm{Sacin}^{*}$ and moment ratios. The specific diffusion- and convection-dependent VI was estimated by Scond and Sacin. Scond was calculated from the phase III slope (SIII) of washout breaths between the 1.5th and 6th lung turnover. Sacin was derived from the first nitrogen SIII and reflects regional acinary ventilation inhomogeneity. LCI5\%, Scond ${ }^{*}$ and $\operatorname{Sacin}^{*}$ were calculated from abbreviated protocols requiring washout until $1 / 20$ th instead of $1 / 40$ th of the initial nitrogen concentration, and the ${ }^{*}$ indices were calculated even earlier 


\section{TABLE 1 Clinical characteristics of primary ciliary dyskinesia patients}

\begin{tabular}{|c|c|}
\hline \multicolumn{2}{|l|}{ General characteristics } \\
\hline Subjects $\mathrm{n}$ & 47 \\
\hline Male/Female $\mathrm{n}$ & $19 / 30$ \\
\hline Age years (mean \pm SD) & $14.7 \pm 6.6$ \\
\hline Situs inversus and/or situs lateralisation defects & $20(41)$ \\
\hline Age at diagnosis years (mean \pm SD) & $6.7 \pm 6.9$ \\
\hline Antibiotic long-term therapy ${ }^{\#}$ & $16(33)$ \\
\hline \multicolumn{2}{|l|}{ Diagnostic } \\
\hline High-frequency-video microscopy available & $49(100)$ \\
\hline Immotile & $25(51)$ \\
\hline Dyskinetic & $23(47)$ \\
\hline Othern & $1(2)$ \\
\hline Transmission electron microscopy available & $39(80)$ \\
\hline Ultrastructural abnormality ${ }^{+}$ & $29(74)$ \\
\hline Normal $l^{\S}$ & $10(26)$ \\
\hline Nasal nitric oxide measurement done & 48 (98) \\
\hline Median (range) $\mathrm{nL} \cdot \mathrm{min}^{-1}$ & $17.1(0.5-303)$ \\
\hline Subjects with level $<77 \mathrm{~nL} \cdot \mathrm{min}^{-1}$ & $40(83)$ \\
\hline Subjects with level $>77 \mathrm{~nL} \cdot \mathrm{min}^{-1 f}$ & $8(17)$ \\
\hline Genetic analysis with PCD defect identified & $15(31)$ \\
\hline \multicolumn{2}{|l|}{ Microbiology at measurement day $\# \#$} \\
\hline Haemophilus influenzae & 9 (19) \\
\hline Staphylococcus aureus & $7(15)$ \\
\hline Others & $4(8)$ \\
\hline Physiological flora & $28(58)$ \\
\hline \multicolumn{2}{|l|}{ Chronic colonisation $^{++}$} \\
\hline Haemophilus influenzae & $15(31)$ \\
\hline Moraxella catarrhalis & $7(14)$ \\
\hline Pseudomonas aeruginosa & $1(2)$ \\
\hline
\end{tabular}

Data are presented as $\mathrm{n}(\%)$, unless otherwise indicated. PCD: primary ciliary dyskinesia. " : antibiotic long-term therapy was defined as more than 6 months of inhaled or oral application in the previous year before measurement; ": this patient showed a normal ciliary beat pattern but a markedly reduced number of motile cilia on respiratory epithelial cells from nasal brushes and in cell culture, transmission electron microscopy showed normal microvilli but severely decreased basal bodies, their nasal nitric oxide was low, their clinical phenotype was severe, and genetic analysis proved a ciliary biogenesis defect with defective centriole generation and reduced multiple motile cilia formation (CCNO defect); ${ }^{+}$: outer dynein arm (ODA) defect $n=5$, combined outer/inner dynein arm (ODA/IDA) defect $n=13$, microtubular disorganisation with central pair/radial spokes defect $n=3$, microtubular disorganisation with IDA defect $n=7$, CCNO defect $n=1 ;{ }^{\S}$ : detailed information on diagnostic results underlying PCD diagnosis is given in the supplementary materials and table E2; ${ }^{f}$ : all eight subjects with nasal nitric oxide above $77 \mathrm{~nL} \cdot \mathrm{min}^{-1}$ presented with a typical clinical phenotype, pathology on chest computed tomography or magnetic resonance imaging, and pathological results in at least two of the three diagnostic procedures of high-frequency-video microscopy, transmission electron microscopy and immunofluorescence; four of these eight subjects had a situs inversus; ${ }^{\# \#}$ : available in 48 patients with PCD; กा: included Moraxella catarrhalis ( $n=2)$ and Klebsiella oxytoca $(n=1)$, Acinetobacter ursingii $(n=1) ;{ }^{++}$: chronic colonisation was defined as the bacterium being detected longer than 1 year.

(useful in severe disease, where the progression of SiII values can form a plateau as early as after the 3rd lung turnover). All gas washout variables are explained in detail in the supplementary material.

\section{Single breath washout}

To assess acinar VI within the timeframe of a single breath, we applied the DTG-SBW as described previously and in detail in the supplementary material [18]. The tracer gas mixture for the DTG-SBW contained $26.3 \%$ helium, $5 \%$ sulfur hexafluoride, $21 \%$ oxygen and balanced nitrogen [10]. The total molar mass of DTG was equivalent to air; therefore, changes in the molar mass signal could be traced back to the tracer gas [18]. The phase III slope was calculated between $65 \%$ and $95 \%$ of the expired tidal volume $[10,17,19]$. Phase III slopes were adjusted for breathing pattern, i.e. tidal volume, as recommended.

\section{Spirometry}

Spirometry was done after inert gas washout tests using the Jaeger MasterScreen Body plethysmograph (CareFusion, Hochberg, Germany) according to ERS/American Thoracic Society (ATS) guidelines [20]. Outcome variables were forced expiratory volume in $1 \mathrm{~s}$ (FEV1) and forced expiratory flow at 25-75\% of forced vital capacity (FEF25-75\%). 


\section{Statistical analysis}

A visual inspection of the data distribution indicated no significant skewing. Washout variables are expressed as mean $\pm \mathrm{SD}$ (range). We calculated z-scores from the healthy controls for inert gas washout outcome variables and from recommended reference equations for spirometry [21]. We only included data from patients with at least two valid measurements after quality control. Upper and lower limits of normal (ULN/ LLN) were defined as $\pm 1.64 \mathrm{z}$-scores [22]. Continuous variables were compared with a t-test. $\mathrm{p}$-values $<0.05$ were considered statistically significant. The results from the agreement of the comparisons from lung function variables were calculated using kappa statistics, for which values $<0.4$ were defined as poor agreement, $0.4-0.6$ as moderate agreement, $>0.6-0.8$ as substantial agreement and $>0.8$ as excellent agreement [23]. We assessed the intra-subject coefficient of variation $(\mathrm{CV}=\mathrm{SD} / \mathrm{mean} \times 100)$ for all gas washout indices in all patients with three valid washout test trials. In patients with two washout test trials, we calculated the variability as the difference between the higher and lower values as a percentage of the higher value; details are given in the supplementary material. Associations between washout variables and spirometry were calculated by linear regression analysis. McNemar's Chi-squared test for binomial proportions for normal and abnormal data was performed to see if inert gas washout indices were more sensitive to VI than FEV1. Analyses were performed using StataTM (Stata Statistical Software: Release 13; StataCorp LP, College Station, TX, USA) and GraphPad Prism (GraphPad Software Inc., La Jolla, CA, USA).

\section{Results}

All 49 patients with PCD and 37 controls were able to perform technically acceptable $\mathrm{N}_{2}-\mathrm{MBW}$ and DTG-SBW, and all 49 patients performed technically acceptable spirometry. The clinical characteristics of the subjects are outlined in table 1, and supplementary tables E1, E2 and E3. Details of the successful tests are included in the supplementary material and supplementary figure E1.

\section{Ability to detect ventilation inhomogeneity}

LCI2.5\% was significantly elevated $(\mathrm{p}<0.001)$ in patients with PCD, with a mean \pm sD (range) of $11 \pm 3.6$ (7.0-23) compared to $6.7 \pm 0.7(5.3-8.0)$ in healthy controls. Other variables of global VI also differed significantly between patients with PCD and healthy controls, including LCI5\% (mean difference 1.8, 95\% CI 1.3-2.3) and $\mathrm{M} 2 / \mathrm{M} 0$ (mean difference 9.0, 95\% CI 5.7-12.4) (both $\mathrm{p}<0.001$ ). Scond was significantly elevated $(\mathrm{p}<0.001)$ in patients with PCD, at $0.053 \pm 0.022(0.005-0.103)$ compared to $0.019 \pm 0.011$ (0.002-0.046) in healthy controls.

Gas washout variables for acinary VI were also significantly elevated in patients with PCD: SIII-DTG was $-0.259 \pm 0.151(-0.690$ to 0.045$)$ in PCD versus $-0.148 \pm 0.097(-0.364$ to -0.005$)$ in controls $(\mathrm{p}<0.001)$. Sacin showed significantly elevated values $(\mathrm{p}=0.002)$ in PCD patients compared to the healthy controls.

Gas washout variables were above the ULN in the majority of patients with PCD (figure 1 and table 2). For LCI $2.5 \%$, 37 out of 47 patients (79\%) had elevated values; for Scond, 36 out of 46 patients (78\%) had elevated values. The respective proportion of patients with abnormal values in alternative washout parameters were as follows: LCI5\% 72\%, Sacin 49\% and SIII-DTG 34\%. In patients with PCD, pathological spirometry results were found in $32 \%$ for $\mathrm{FEV}_{1}$ and in $32 \%$ for $\mathrm{FEF}_{2} 5-75 \%$. The mean $\pm \mathrm{SD}$ of $\mathrm{FEV}_{1}$ was $-1.1 \pm 1.6$ in $\mathrm{z}$-scores for patients with PCD. Including only patients with complete data or using an ULN/LLN of \pm 1.96 SD resulted in comparable findings (table 2). Tidal volume was larger in controls than in PCD patients but the difference was not statistically significant. Mean \pm SD tidal volume was $615 \pm 31 \mathrm{~mL}$ in controls compared to $536 \pm 27 \mathrm{~mL}$ in PCD patients $(\mathrm{p}=0.06)$. Calculation with McNemar's Chi-squared test revealed that $\mathrm{LCI}_{5} \%, \mathrm{M}_{2} / \mathrm{M} 0, \mathrm{M}_{1} / \mathrm{M} 0$, Scond and Sacin were more sensitive in detecting VI than FEV1 $(\mathrm{p}<0.05)$. Sacin $^{*}$ and SIII-DTG were comparably sensitive to FEV1.

\section{Duration of gas washout measurements}

LCI2.5\% measurement duration was the longest at 19.8 $\pm 11.2 \mathrm{~min}$ (5-61 min). As expected, several alternative outcome variables required less time. Scond and Sacin required on average $8.6 \pm 2.3 \mathrm{~min}$ (5-14 min), LCI5\% required $10.8 \pm 4.6 \mathrm{~min}(4.3-22.6 \mathrm{~min})$ and SIII-DTG only $5 \mathrm{~min}$ for three measurements (table 2).

\section{Intra-test variability of the different lung function indices}

In general, variability was similar in those patients who achieved two trials and those with three trials. The variability of indices derived from areas under the curve (moment ratios, LCI) was low, ranging between $4 \%$ and $10 \%$ and comparable between PCD patients and controls. In contrast, the variability of indices derived from phase III slopes (Sacin, Scond, SIII-DTG) ranged between $24 \%$ and $76 \%$ across both groups and was systematically higher in controls compared to PCD (supplementary table E4). For most variables, the CV tended to be lower in patients with PCD than in healthy controls (supplementary table E4). Similar results were found for the group of 30 patients with PCD with only two valid trials (supplementary table E5). 


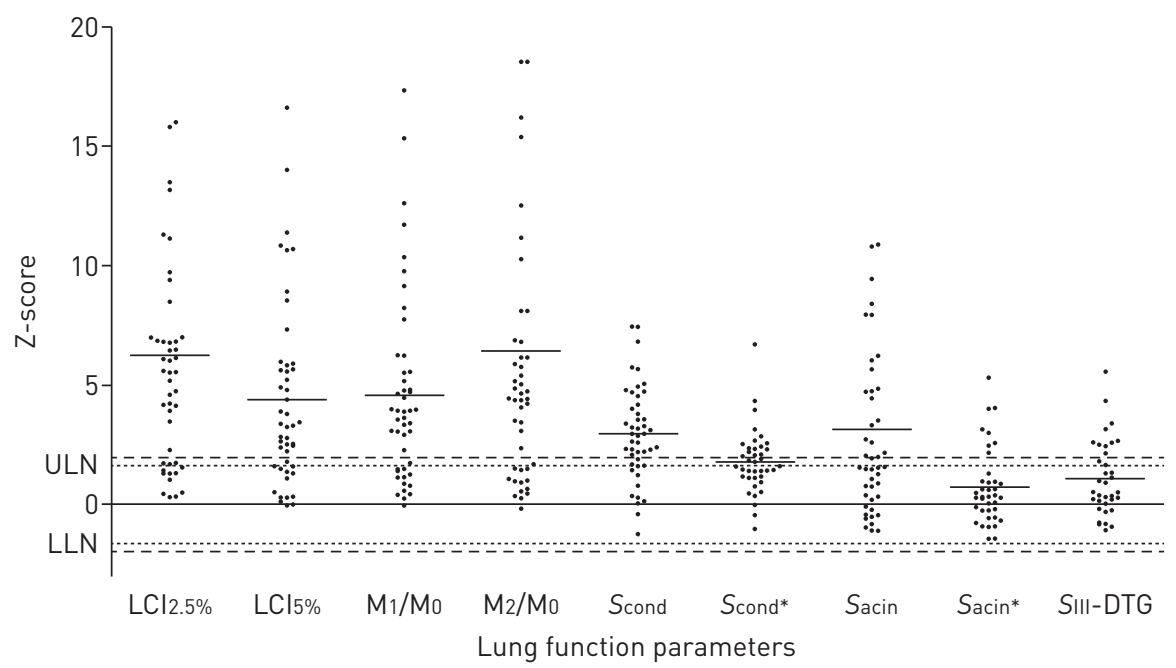

FIGURE 1 Illustration of all gas washout outcome variables. Lung function values $L C l 2.5 \%, L C l 5 \%, M_{1} / M_{0}, M_{2} /$ Mo, Scond, Sacin, Scond*, Sacin* and SIII-DTG are given in z-scores. For an explanation of these terms, see the supplementary material. Narrow dashed lines indicate upper and lower limits of normal (ULN/LLN) ( $\pm 1.64 \mathrm{z}$-scores). Longer dashed lines indicate ULN/LLN ( $\pm 1.96 \mathrm{z}$-scores). Individual mean values of the indices are marked as a line. For better illustration, values of more than $20 \mathrm{z}$-scores are not shown: one value for Sacin (21.4 z-score) and two values for both $\mathrm{LCl} 2.5 \%$ (23.7; $20.4 \mathrm{z}$-scores) and M2/Mo (28.4; 35.6 z-scores) were removed. SIII-DTG values are transformed from negative to positive values.

Agreement between lung function variables and $\mathrm{LCl} 2.5 \%$ to detect ventilation inhomogeneity Using kappa statistics, we found poor agreement in detecting abnormal lung function (kappa coefficient=0.4) between Scond and LCI2.5\%. Agreement between LCI5\%, M1/M0 and M2/M0, respectively, with LCI $2.5 \%$ in detecting abnormal lung function was substantial, with a kappa coefficient between 0.6 and 0.7 (table 2).

Association between $\mathrm{LCl} 2.5 \%$, shorter gas washout outcomes and spirometry

We found a good association between $\mathrm{LCI}_{2.5 \%}$ and $S_{\text {cond }}{ }^{*}\left(\mathrm{R}^{2}=0.47, \mathrm{p}<0.001\right)$ but not between LCI $2.5 \%$ and Scond $\left(\mathrm{R}^{2}=0.03, \mathrm{p}=0.238\right)$, see figure 2 and supplementary figure E2. Significant associations with $\mathrm{LCI} 2.5 \%$

\section{TABLE 2 Outcome variables of lung function measurements in patients with primary ciliary dyskinesia}

\begin{tabular}{|c|c|c|c|c|c|}
\hline Indices & $z$-score (mean $\pm s D$ ) & \multicolumn{2}{|c|}{ Pathological values ${ }^{\#}$} & $\begin{array}{l}\text { Agreement with } \\
\mathrm{LCl}^{2.5 \%}{ }^{+} \mathrm{k}(95 \% \mathrm{CI})\end{array}$ & Duration ${ }^{\S} \min$ \\
\hline LCl $2.5 \%$ & $6.3 \pm 5.2$ & $37 / 47$ (79) & $25 / 33(76)$ & N/A & $19.8 \pm 11.2(5-61)$ \\
\hline LCl5\% & $4.4 \pm 3.9$ & $34 / 47$ (72) & $25 / 33(76)$ & $0.7(0.5-0.9)$ & $10.8 \pm 4.6(4.3-22.6)$ \\
\hline $\mathrm{M}_{1} / \mathrm{M}_{0}$ & $6.4 \pm 7.2$ & $34 / 47$ (72) & $26 / 33(79)$ & $0.6(0.3-0.9)$ & $\mathrm{N} / \mathrm{A}$ \\
\hline Scond* & $1.8 \pm 1.4$ & $19 / 38(50)$ & 18/33 (55) & $0.5(0.236-0.7)$ & $4.3 \pm 1.2(2.3-7)$ \\
\hline Sacin & $3.1 \pm 4.3$ & $22 / 45$ (49) & $17 / 33(52)$ & $0.2(-0.013-0.4)$ & $8.6 \pm 2.3(4.5-14.2)$ \\
\hline Sacin* & $0.8 \pm 1.6$ & $8 / 37(21.6)$ & $8 / 33(24)$ & $0.2(0.03-0.3)$ & $4.3 \pm 1.2(2.3-7)$ \\
\hline SIII-DTG & $-1.1 \pm 1.6$ & $12 / 35(34)$ & $12 / 33(36)$ & $0.3(0.103-0.5)$ & $5^{f}$ \\
\hline FEV $_{1}$ & $-1.1 \pm 1.6$ & $15 / 47$ (32) & $10 / 33(30)$ & $0.2(-0.004-0.3)$ & $\mathrm{N} / \mathrm{A}$ \\
\hline FEF $25-75 \%$ & $-1.1 \pm 1.9$ & $15 / 47$ (32) & $10 / 33(30)$ & $0.2(0.08-0.4)$ & $\mathrm{N} / \mathrm{A}$ \\
\hline
\end{tabular}

Upper and lower limits of normal (ULN, LLN) were defined as \pm 1.64 z-scores. Using an ULN/LLN of $\pm 1.96 \times$ SD resulted in comparable findings (LCl2.5\% 34/47 (72\%); LCl5\% 33/47 (70\%), Scond 33/46 (72\%), Sacin 21/45 (47\%) and FEV1 11/47 (23\%)). LCl2.5\%: lung clearance index 2.5\%; LCl5\%; lung clearance index 5\%; M1/Mo, M2/M0: moment ratio; Scond/Sacin, Scond*/Sacin* and SIII-DTG: normalised phase III slope indices /see text for explanation); FEV1: forced expiratory volume in $1 \mathrm{~s}$; FEF25-75\%: forced expiratory flow at $25-75 \%$ of forced vital capacity; N/A: not available/ applicable. \#: data for the pathological variables are presented as the number of patients with pathological values/number of patients that performed the test (\%); ": calculation of pathological values using only measurements where all indices were of good quality and no indices had to be excluded for technical or quality control reasons; ${ }^{+}$: test agreement is presented as kappa $\left(95 \%\right.$ CI); ${ }^{\S}$ : test duration for three trials with two pauses is given in mean \pm SD (range); ${ }^{f}$ : one test (10 breaths) was estimated as $60 \mathrm{~s}$ to account for complete expiration of gas. 
were found for LCI5\% $\left(\mathrm{R}^{2}=0.8\right)$ and SIII-DTG $\left(\mathrm{R}^{2}=0.4\right)$, as shown in figure 3 and supplementary figure E3. A significant association was found between $S$ cond and $S_{\text {cond }}{ }^{*}\left(R^{2}=0.3, p<0.001\right)$ and between $S$ acin and $\operatorname{Sacin}^{*}\left(\mathrm{R}^{2}=0.7, \mathrm{p}<0.001\right)$.

LCI $2.5 \%$ was negatively associated with FEF $25-75 \%\left(\mathrm{R}^{2}=0.5, \mathrm{p}<0.001\right)$ and FEV1 $\left(\mathrm{R}^{2=} 0.4, \mathrm{p}<0.001\right)$. SIII-DTG was associated with $F E F 25-75 \%\left(R^{2}=0.2, p<0.001\right)$ and FEV1 $\left(R^{2}=0.18, p=0.012\right)$. Sacin was negatively associated with FEF25-75\% and FEV1, while Scond was not associated with FEV1 or FEF25-75\%, as outlined in table 3 and supplementary table E6.

\section{Discussion}

Main findings

We confirm results from previous studies showing a high prevalence of VI in PCD lung disease. In this study we show for the first time that global and convection VI can be estimated not only by LCI $2.5 \%$, but also by alternative washout variables like the LCI5\%, moment ratio and Scond. Compared to LCI2.5\%, LCI5\% and Scond seem more practical for routine application because measurement duration is much shorter. These three gas washout variables seem to be more sensitive in detecting pathological lung function than spirometry; however, physiological differences between outcome variables can account for differences in duration, sensitivity and intra-subject variability. Overall, our findings suggest that new outcome variables, e.g. LCI5\%, moment ratio and Scond, might be promising outcome measures to detect global and convection-dependent VI in routine clinical practice in patients with PCD.

\section{Comparison with previous studies}

Sensitive lung function techniques are considered the current gold standard to assess early VI arising in small airways. In line with previous literature, we have shown that LCI5\% is as sensitive as standard LCI2.5\%, and requires less time in patients with PCD [24]. In our results, using a nitrogen washout cut-off of $5 \%$ reduced the time needed to perform three washout tests to half that required for a cut-off of $2.5 \%$, without reducing the sensitivity. Moment ratio and LCI5\% were well associated with LCI $2.5 \%$ and therefore could be promising time-saving alternatives. For this study, we have considered LCI $2.5 \%$ as the gold standard, yet there is no consensus as to how VI should be best assessed in patients with PCD.

We also report for the first time data on abbreviated phase III slope indices in PCD. However, lower sensitivity for abnormal lung function and higher intra-test variability compared to LCI $2.5 \%$ constrain their current applicability as an alternative to LCI2.5\% in mild to moderate PCD lung disease.

Previous studies in non-CF bronchiectasis patients showed that LCI $2.5 \%$ and Scond were significantly elevated compared to healthy controls [25], similar to the findings in PCD patients in our present study. We assume that PCD lung disease primarily affects small convection-dependent airways. This is supported by the high prevalence of abnormal Scond found in this and previous studies of patients with PCD [3]. Furthermore, the poor agreement resembling a lack of association between LCI $2.5 \%$ and Scond in our and a previous study [2] suggests that Scond and LCI measure VI arising at different sites in the airways. Scond might be more susceptible to an invariable breathing pattern. This could explain why LCI and moment ratio seem to be more strongly associated with structural changes in HRCT than Scond [3]. In the latter study, HRCT scan and lung function were not performed on the same day, which could also have influenced structure-function relations. The association between VI indices and structural changes also

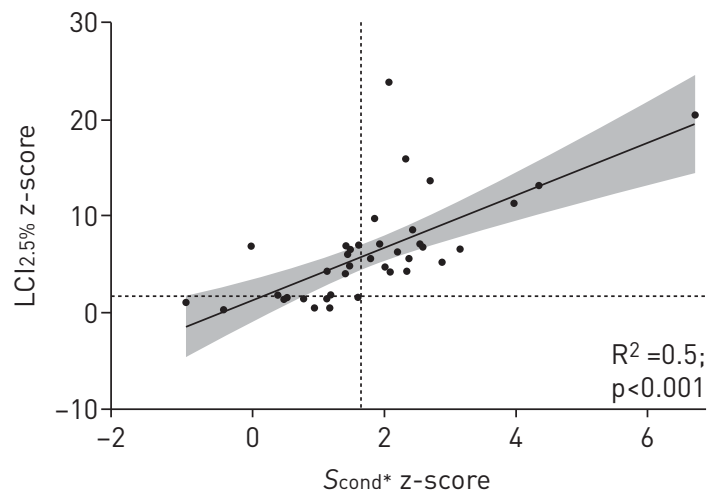

FIGURE 2 Association between LCl2.5\% and Scond* in 37 patients (black points) with primary ciliary dyskinesia, given as z-scores. Fitted values are shown by the black line. The grey background denotes the $95 \%$ confidence interval. Dashed lines indicate the upper and lower limits of normal $( \pm 1.64 \mathrm{z}$-scores). 


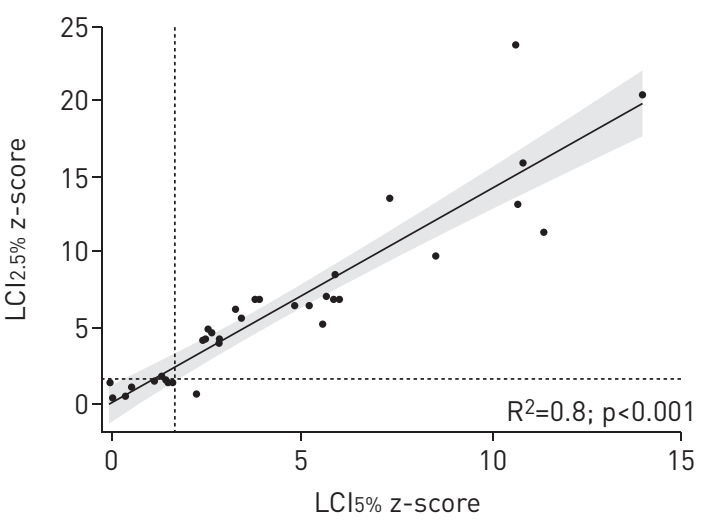

FIGURE 3 Association between LCl2.5\% and LCl5\% in 47 patients (black points) with primary ciliary dyskinesia, given as z-scores. Fitted values are shown by the black line. The grey background denotes the $95 \%$ confidence interval. Dashed lines indicate the upper and lower limits of normal $( \pm 1.64 z$-scores).

seems to be influenced by the tracer gas in use $[3,26]$. Hence it seems sensible to determine both LCI2.5\%, and LCI5\% as well as Scond and moment ratio in order to comprehensively study lung function impairment in PCD. Variation in the disease phenotype may require different tests to detect specific types of pulmonary impairment [27].

In this study, we report for the first time data on abbreviated phase III slope indices (Scond ${ }^{*}$ and $\left.S_{\text {acin }}{ }^{*}\right)$ in patients with PCD. We did not find an association between Scond and FEV1 or LCI2.5\%, but we did find an association between $S_{\text {cond }}{ }^{*}$ and FEV1 and LCI2.5\% (supplementary figures E4 and E5). Assessing Scond ${ }^{\star}$ and $S_{\text {acin }}{ }^{*}$ might be sensible because the Scond calculation is distorted in patients affected by severe lung disease by the early flattening of phase III slopes after lung turnovers three to four, and Sacin, the first breath's phase III slope, is already maximally elevated at this point [28]. Therefore, abbreviated phase III slope indices might be of use to maintain sensitivity in detecting VI over a broad range of disease severity, in addition to their advantage of being shorter tests. Furthermore, our findings showed that Scond ${ }^{*}$ was positively associated with LCI $2.5 \%$ and spirometry, whereas Scond was not associated with any of them. Interestingly, other groups have found an association between LCI2.5\% and Scond ${ }^{*}$ in CF, which supports the physiological plausibility of our findings in PCD [29]. We assume that Scond ${ }^{\star}$ resembles fast-ventilated lung compartments of the lung. These compartments are thought to contribute mostly to the early phases of the MBW and to determine the magnitude of LCI and $S$ cond $^{*}$ [30]. Further studies are needed to examine if this finding is due to mathematical or physiological issues.

In our study, Sacin was moderately sensitive in detecting VI. A previous study showed a good association between Sacin and HRCT [3]. In the present study, the DTG-SBW was less sensitive than Scond and

\begin{tabular}{|c|c|c|c|c|}
\hline Indices & Coefficient & $95 \% \mathrm{Cl}$ & $\mathbf{R}^{2}$ & $\mathrm{p}$-value \\
\hline $\mathrm{LCl}_{2.5 \%}$ & -0.2 & -0.3 to -0.1 & 0.4 & $<0.001$ \\
\hline LCl5\% & -0.3 & -0.4 to -0.2 & 0.5 & $<0.001$ \\
\hline $\mathrm{M}_{1} / \mathrm{M}_{0}$ & -0.3 & -0.4 to -0.2 & 0.5 & $<0.001$ \\
\hline $\mathrm{M}_{2} / \mathrm{Mo}_{0}$ & -0.1 & -0.19 to -0.08 & 0.4 & $<0.001$ \\
\hline Scond & -0.2 & -0.4 to 0.04 & 0.06 & 0.107 \\
\hline Scond* & -0.7 & -1.0 to -0.5 & 0.4 & $<0.001$ \\
\hline Sacin & -0.2 & -0.3 to -0.06 & 0.2 & 0.003 \\
\hline Sacin* & -0.3 & -0.6 to 0.1 & 0.1 & 0.055 \\
\hline SIII-DTG & 0.4 & 0.1 to 0.8 & 0.18 & 0.012 \\
\hline
\end{tabular}

Association between $\mathrm{FEV} 1$ and different gas washout indices: $\mathrm{LCl}_{2.5 \%} \mathrm{LCl} 5 \%, \mathrm{M}_{1} / \mathrm{M}_{0}, \mathrm{M}_{2} / \mathrm{M}_{0}, \mathrm{Scond} / \mathrm{Sacin}_{\text {, }}$ Scond*/Sacin* and SIII-DTG, normalised phase III slope indices (see text for explanation). Significant values are marked in bold. Results are derived from linear regression analysis. Significant associations were found between $\mathrm{LCl}_{2.5 \%}$ and $\mathrm{LCl} 5 \%$ (coefficient: $1.2,95 \% \mathrm{Cl} 1.08-1.4, \mathrm{p}<0.001, \mathrm{R}^{2}=0.8$ ) and with SIII-DTG (coefficient: $-2.2,95 \% \mathrm{Cl}-3.2$ to $-1.2, \mathrm{p}<0.001, \mathrm{R}^{2}=0.4$ ). $\mathrm{FEV}$ : forced expiratory volume in $1 \mathrm{~s}$; $\mathrm{LCl} 2.5 \%$ : lung clearance index 2.5\%; LCl5\%: lung clearance index 5\%; M1/Mo, M2/Mo: moment ratio; Scond/Sacin, Scond*/Sacin*, SIII-DTG: normalised phase III slope indices (see text for explanation). 
LCI $2.5 \%$ in patients with PCD. As a conclusion, in contrast with other chronic lung diseases such as COPD and asthma, diffusion-dependent VI seems less affected in PCD. Whether the SIII-DTG might be more useful in patients with a certain phenotype is still unclear [31]. In line with previous observations, FEF25-75\% did not add to the interpretation of spirometry in PCD patients, because $\mathrm{FEF} 25-75 \%$ and $\mathrm{FEV} 1$ were similarly discriminative [2]. The $\mathrm{M}_{1} / \mathrm{M}_{0}$ was moderately associated with FEV1 and previous studies have demonstrated the ability of $\mathrm{M}_{1} / \mathrm{M}_{0}$ to sensitively measure treatment responses in $\mathrm{CF}$ [32], which shows that this parameter could also be promising in patients with PCD.

We assume that different results on the association and sensitivity of washout parameters in this study could be caused by the diversity of functional, ultrastructural and genetic defects in PCD; different washout procedures applied; or differences in the age of the patients tested. Longitudinal studies will be necessary to show the variability, long-term repeatability and sensitivity of the different gas washout indices [33] for interventions such as inhalation or antibiotic therapy.

\section{Strengths and limitations}

Taking into account that PCD is a rare lung disease with an approximate prevalence of 1 in 10000 [34], we were able to include a relative large number of PCD patients in this study.

PCD diagnosis relies on actual recommendations, and the study population showed clinical characteristics typical of a mainly paediatric PCD cohort. Not surprisingly, our findings are in line with previous studies, which found elevated values for Scond and LCI2.5\% in patients with PCD [2, 3]. All data from the current study were obtained using the same equipment, software and protocols. Quality control and analysis were done by the same person (SN) for all measurements.

We could not perform the DTG-SBW in all patients owing to the fact that the manufacturer delivered incorrect gas mixtures at a certain time period. However, compared to other studies, the number of patients with PCD who performed the DTG-SBW remained high, and allowed meaningful interpretation $[9,19]$. Healthy controls were assessed at a single centre only and their age distribution differed slightly from that of the patients. However, 1) adjusting for height or age did not change the results of our analysis, 2) published normal values measured by the same equipment are similar to ours [3] and 3) measurement differences in healthy controls between centres are expected to be far lower than differences between patients with PCD and controls. Most important, our findings do not depend upon absolute values of controls.

In conclusion, this study suggest that LCI5\%, moment ratio and Scond might be promising alternatives to LCI $2.5 \%$ for monitoring lung function in routine clinical practice in patients with PCD, despite the high variability of some of these parameters. We suggest that LCI5\%, moment ratio and Scond are outcome variables that can be measured during tidal breathing in a much shorter time than LCI2.5\%, and that they have a high sensitivity to detect VI compared to all other gas washout variables and spirometry. Whether they only provide a more comprehensive picture of respiratory impairment in PCD, or if they yield additional physiological information that is helpful from a clinical perspective, needs to be determined in future studies.

\section{Acknowledgements}

The authors would like to thank all children and their families for their participation in the study. The authors would like to express their thanks to all the study nurses for their patient care, support in lung function measurement and recruitment of the children.

Authors contributions: S. Nyilas, A. Schlegtendal, P. Latzin and C. Koerner-Rettberg designed the study concept. S. Nyilas and A. Schlegtendal collected the study data. S. Nyilas analysed the data. S. Nyilas, A. Schlegtendal, F. Singer, M. Goutaki, C.E. Kuehni, C. Casaulta, P. Latzin and C. Koerner-Rettberg interpreted the data. S. Nyilas drafted the manuscript. All authors revised the manuscript.

\section{References}

1 Madsen A, Green K, Buchvald F, et al. Aerobic fitness in children and young adults with primary ciliary dyskinesia. PloS One 2013; 8: e71409.

2 Green K, Buchvald FF, Marthin JK, et al. Ventilation inhomogeneity in children with primary ciliary dyskinesia. Thorax 2012; 67: 49-53.

3 Boon M, Vermeulen FL, Gysemans W, et al. Lung structure-function correlation in patients with primary ciliary dyskinesia. Thorax 2015; 70: 339-345.

4 Barbato A, Frischer T, Kuehni CE, et al. Primary ciliary dyskinesia: a consensus statement on diagnostic and treatment approaches in children. Eur Respir J 2009; 34: 1264-1276.

5 Singer F, Kieninger E, Abbas C, et al. Practicability of nitrogen multiple-breath washout measurements in a pediatric cystic fibrosis outpatient setting. Pediatr Pulmonol 2013; 48: 739-746.

6 Yammine S, Singer F, Gustafsson P, et al. Impact of different breathing protocols on multiple-breath washout outcomes in children. J Cyst Fibros 2014; 13: 190-197.

7 Ahmad F, Irving S, Alton E, et al. Multiple breath washouts in children can be shortened without compromising quality. Eur Respir J 2015; 46: 1814-1816. 
8 Husemann K, Berg N, Engel J, et al. Double tracer gas single-breath washout: reproducibility in healthy subjects and COPD. Eur Respir J 2014; 44: 1210-1222.

9 Singer F, Abbas C, Yammine S, et al. Abnormal small airways function in children with mild asthma. Chest 2014; 145: 492-499.

10 Singer F, Stern G, Thamrin C, et al. A new double-tracer gas single-breath washout to assess early cystic fibrosis lung disease. Eur Respir J 2013; 41: 339-345.

11 Thompson BR, Douglass JA, Ellis MJ, et al. Peripheral lung function in patients with stable and unstable asthma. J Allergy Clin Immunol 2013; 131: 1322-1328.

12 Vanderhelst E, De Meirleir L, Schuermans D, et al. Evidence of an acinar response following treatment for exacerbation in adult patients with cystic fibrosis. Respiration 2014; 87: 492-498.

13 Verbanck S, Schuermans D, Van Muylem A, et al. Conductive and acinar lung-zone contributions to ventilation inhomogeneity in COPD. Am J Respir Crit Care Med 1998; 1573-1577.

14 Neesen J, Kirschner R, Ochs $\mathrm{M}$, et al. Disruption of an inner arm dynein heavy chain gene results in asthenozoospermia and reduced ciliary beat frequency. Hum Mol Genet 2001; 10: 1117-1128.

15 Nüßlein T, Brinkmann F, Ahrens P, et al. Diagnostik der primären ziliären Dyskinesie [Diagnostics of primary ciliary dyskinesia]. Monatsschr Kinderheilkd 2013; 161: 406-416.

16 Singer F, Houltz B, Latzin P, et al. A realistic validation study of a new nitrogen multiple-breath washout system. PloS One 2012; 7: e36083.

17 Robinson PD, Latzin P, Verbanck S, et al. Consensus statement for inert gas washout measurement using multiple- and single-breath tests. Eur Respir J 2013; 41: 507-522.

18 Singer F, Stern G, Thamrin C, et al. Tidal volume single breath washout of two tracer gases--a practical and promising lung function test. PloS One 2011; 6: e17588.

19 Abbas C, Singer F, Yammine S, et al. Treatment response of airway clearance assessed by single-breath washout in children with cystic fibrosis. J Cyst Fibros 2013; 12: 567-574.

20 Miller MR, Hankinson J, Brusasco V, et al. Standardisation of spirometry. Eur Respir J 2005; 26: 319-338

21 Stanojevic S, Wade A, Cole TJ, et al. Spirometry centile charts for young Caucasian children: the Asthma UK Collaborative Initiative. Am J Respir Crit Care Med 2009; 180: 547-552.

22 Quanjer PH, Stanojevic S, Cole TJ, et al. Multi-ethnic reference values for spirometry for the 3-95-yr age range: the global lung function 2012 equations. Eur Respir J 2012; 40: 1324-1343.

23 Goh NS, Desai SR, Veeraraghavan S, et al. Interstitial lung disease in systemic sclerosis: a simple staging system. Am J Respir Crit Care Med 2008; 177: 1248-1254.

24 Green K, Ejlertsen JS, Madsen A, et al. Abbreviation modalities of nitrogen multiple-breath washout tests in school children with obstructed lung disease. Pediatr Pulmonol 2015; 51: 624-632.

25 Gonem S, Scadding A, Soares M, et al. Lung clearance index in adults with non-cystic fibrosis bronchiectasis. Respir Res 2014; 15: 59.

26 Irving SJ, Ives A, Davies G, et al. Lung clearance index and high-resolution computed tomography scores in primary ciliary dyskinesia. Am J Respir Crit Care Med 2013; 188: 545-549.

27 Noone PG, Leigh MW, Sannuti A, et al. Primary ciliary dyskinesia: diagnostic and phenotypic features. Am J Respir Crit Care Med 2004; 169: 459-467.

28 Verbanck S, Paiva M, Schuermans D, et al. Acinar and conductive ventilation heterogeneity in severe CF lung disease: back to the model. Respir Physiol Neurobiol 2013; 188: 124-132.

29 Horsley AR, Macleod KA, Robson AG, et al. Effects of cystic fibrosis lung disease on gas mixing indices derived from alveolar slope analysis. Respir Physiol Neurobiol 2008; 162: 197-203.

30 Gustafsson PM, Robinson PD, Gilljam M, et al. Slow and fast lung compartments in cystic fibrosis measured by nitrogen multiple-breath washout. J Appl Physiol 2014; 117: 720-729.

31 Nyilas S, Singer F, Kumar N, et al. Physiological phenotyping of paediatric chronic obstructive airway diseases. J Appl Physiol 2016; 121: 324-332.

32 Stanojevic S, Jensen R, Sundaralingam D, et al. Alternative outcomes for the multiple breath washout in children with CF. J Cyst Fibros 2015; 14: 490-496.

33 Bush A, Irving S. Wavering in the breeze: is multiple breath washout useful in primary ciliary dyskinesia? Thorax 2015; 70: 305-306.

34 Praveen K, Davis EE, Katsanis N. Unique among ciliopathies: primary ciliary dyskinesia, a motile cilia disorder. F1000Prime Rep 2015; 7: 36 